Sādhanā Vol. 36, Part 2, April 2011, pp. 209-221. (C) Indian Academy of Sciences

\title{
Thermo elastic waves with thermal relaxation in isotropic micropolar plate
}

\author{
SOUMEN SHAW ${ }^{1, *}$ and BASUDEB MUKHOPADHYAY ${ }^{2}$ \\ ${ }^{1}$ Department of Mathematics, Meghnad Saha Institute of Technology, Kolkata, \\ West Bengal 711103, India \\ ${ }^{2}$ Department of Mathematics, Bengal Engineering and Science University, Shibpur, \\ West Bengal 711103, India \\ e-mail: shaw_soumen@rediffmail.com; bmukharjee2006@yahoo.co.in
}

MS received 15 March 2010; revised 22 December 2010; accepted 19 January 2011

\begin{abstract}
In the present investigation, we have discussed about the features of waves in different modes of wave propagation in an infinitely long thermoelastic, isotropic micropolar plate, when the generalized theory of Lord-Shulman $(\mathrm{L}-\mathrm{S})$ is considered. A more general dispersion equation is obtained. The different analytic expressions in symmetric and anti-symmetric vibration for short as well as long waves are obtained in different regions of phase velocities. It is found that results agree with that of the existing results predicted by Sharma and Eringen in the context of various theories of classical as well as micropolar thermoelasticity.
\end{abstract}

Keywords. Micropolar; generalized thermoelasticity; symmetric and anti-symmetric mode.

\section{Introduction}

It is well-known that when a wave propagating into a material body, its motion and the deformation of the material body depends on its inner structure. Not only its inner structure but also depends on the motions of the inner structures. Classical elasticity ignores this effect by ascribing only transformation degrees of freedom of the material point of the body. The micropolar theory of Eringen (1965) describes an intrinsic rotation of the microstructures and developed a model that can support the body and surface couples and provide a high frequency optical branch of wave spectrum.

In classical coupled thermoelasticity, the heat conduction equation, which is based on classical Fourier's law, is a parabolic type partial differential equation and it allows an infinite speed of thermal signals. Generalized thermoelasticity, in which the heat conduction equation is a hyperbolic type, eliminates the paradox of infinite heat propagation speed.

Based on the theory made by Cattanco (1958), which is a generalized version of Fourier's law, Kaliski (1965) obtained the generalized version of coupled thermoelasticity in anisotropic

*For correspondence 
elastic media. In isotropic elastic media Lord \& Shulman (1967), Dhaliwal \& Sherief (1980) deduced independently the generalized version of classical coupled theory. In the above theories flux rate term is incorporated in Fourier's law of heat conduction equation. The new theories proposed by the authors are referred to as thermoelasticity with thermal relaxation or extended thermoelasticity.

Another generalization of thermoelasticity is considered first by Muller (1967, 1971), which includes the temperature rate among the constitutive equations. Chowdhury et al (1979), Chowdhury \& Glockner (1979) have obtained constitutive equations for thermorigid dielectrics and thermoelastic dielectrics respectively by employing Muller's approach. Based on entropy production in equality, proposed by Green \& Laws (1972), a more explicit and simpler theory than Muller is formulated by Green \& Lindsay (1972). A remarkable feature of Green and Lindsay theory is that, it does not violate the Classical Fourier's law, if the material has a centre of symmetry at each point. Independent work in this area is done by Suhubi (1982). The theories considered above are referred to as temperature rate dependent thermoelasticity and it includes two relaxation times.

The thermoelasticity without energy dissipation is developed by Green \& Nagdi (1993). A uniqueness theorem in thermoelasticity is considered by Chandrasekharaiah \& Debnath (1988).

A number of research workers used the above mentioned theories of generalized thermoelasticity in various wave propagation problems in infinite and semi-infinite elastic media, out of which we can mention the name of Chandrasekharaiah \& Debnath (1988), Ezzat et al (2002), McCarthy (1972), Sharma (2001), Rakshit \& Mukhopadhyay (2005, 2007), Mukhopadhyay \& Bera (1992), Das et al (1997). Valuable information and developments in thermoelasticity and wave propagation are available in the books of Nowacki (1962), Nowinski (1978), Boley \& Weiner (1960), Eringen \& Maugin (1990), Graff (1991), etc. A compact idea in generalized thermoelasticity can be generated from the review work of Chandrasekharaiah (1986).

Theories of micropolar elastic solids in which the micromotions of the particle contained in a macrovolume element with respect to its centroid are considered, is introduced by Eringen (1966, 1973), Eringen \& Suhubi (1964), Suhubi \& Eringen (1964). Eringen, who is the pioneer in the field of microcontinuum, did a lot of research work in the development of micropolar elasticity in last few decades. It seems relevant to mention the important and valuable works by Nowacki (1975, 1970), Iesan (1967), Erbay \& Suhubi (1989), Rao (1988), Tomar et al (1998) in this field.

Singh \& Kumar (1998), Othman \& Singh (2007), Kumar \& Ailawalia (2006, 2007), Kumar \& Kansal (2008), Kumar \& Partap (2006) considered various problems in micropolar thermoelastic solids using generalized theory of thermoelasticity.

The present paper is concerned with a micropolar thermoelastic problem in an infinite plate with insulated as well as isothermal boundary conditions in the context of generalized theory of thermoelasticity due to Lord \& Shulman (1967).

\section{Basic equations}

The basic governing equations in generalized isotropic micropolar thermoelasticity without heat sources and body forces are given by

$$
\begin{gathered}
(\lambda+2 \mu+\kappa) \vec{\nabla}(\vec{\nabla} \cdot \vec{u})-(\mu+\kappa) \vec{\nabla} \times \vec{\nabla} \times \vec{u}+\kappa \vec{\nabla} \times \vec{\phi}-\beta_{0} \vec{\nabla} \theta=\rho \ddot{\vec{u}} \\
(\alpha+\beta+\gamma) \vec{\nabla}(\vec{\nabla} \cdot \vec{\phi})-\gamma \vec{\nabla} \times \vec{\nabla} \times \vec{\phi}+\kappa \vec{\nabla} \times \vec{u}-2 \kappa \vec{\phi}=\rho j \ddot{\bar{\phi}}
\end{gathered}
$$


where $\alpha, \beta, \gamma, \lambda, \mu, \kappa$ are the elastic constants, $\rho$ is the density of the material per unit mass, $\beta_{0}=(3 \lambda+2 \mu+\kappa) \alpha_{t}, \alpha_{t}$ is the co-efficient of linear thermal expansion, $j$ is the microinertia and $\vec{u}, \vec{\varphi}$ are the displacement and micro-rotation vectors of the elastic body respectively.

The heat conduction equation given by Lord \& Shulman (1967) is

$$
K \nabla^{2} \theta=\left(1+\tau \frac{\partial}{\partial t}\right) \rho C_{e} \dot{\theta}+\beta_{0} \theta_{0}\left(1+\tau \frac{\partial}{\partial t}\right) \frac{\partial}{\partial t}(\vec{\nabla} \cdot \vec{u}),
$$

where $\theta$ is the temperature above the initial temperature $\theta_{0}, \tau$ represent the thermal relaxation time, $C_{e}$ is the specific heat at constant strain.

\section{Formulation of the problem}

The present problem is concerned about the wave propagation in an infinitely long plate along $x_{1}$-axis bounded by the surfaces $x_{2}= \pm h$ (h is very small) so that the problem reduces to a problem of plane strain.

In the plane strain parallel to $x_{1} x_{2}$ plane, we consider

$$
u_{3}=0, \phi_{1}=\phi_{2}=0 \text { and } \vec{u}=\left(u_{1}, u_{2}, 0\right), \vec{\phi}=(0,0, \phi) .
$$

From the equations (2.1) to (2.3) we obtain the following equations in dimensionless form by omitting the primes,

$$
\begin{gathered}
u_{1,11}+\left(1-\delta^{2}\right) u_{2,12}+\delta^{2} u_{1,22}+\phi_{, 2}-\theta_{, 1}=\ddot{u}_{1} \\
u_{2,22}+\left(1-\delta^{2}\right) u_{1,12}+\delta^{2} u_{2,11}-\phi_{, 1}-\theta_{, 2}=\ddot{u}_{2} \\
\frac{\gamma \omega^{* 2}}{\kappa c_{1}^{2}} \nabla^{2} \phi+\frac{\kappa}{\rho c_{1}^{2}}\left(u_{2,1}-u_{1,2}\right)-2 \phi=\frac{\omega^{* 2} \rho j}{\kappa} \ddot{\phi} \\
\nabla^{2} \theta=\left(1+\tau \frac{\partial}{\partial t}\right) \dot{\theta}+\varepsilon\left(1+\tau \frac{\partial}{\partial t}\right)\left(\dot{u}_{1,1}+\dot{u}_{2,2}\right),
\end{gathered}
$$

where,

$$
x_{i}^{\prime}=\frac{\omega^{*}}{c_{1}} x_{i}, u_{i}^{\prime}=\frac{\omega^{*} \rho c_{1}}{\beta_{0} \theta_{0}} u_{i}, \tau^{\prime}=\omega^{*} \tau, \theta^{\prime}=\frac{\theta}{\theta_{0}}, t^{\prime}=\omega^{*} t, \phi^{\prime}=\frac{\kappa}{\beta_{0} \theta_{0}} \phi,
$$

$h_{1}^{\prime}=\frac{h_{1} c_{1}}{\omega^{*}}, i=1,2$ are the dimensionless variables, in which

$$
c_{1}^{2}=\frac{\lambda+2 \mu+\kappa}{\rho}, c_{2}^{2}=\frac{\mu+\kappa}{\rho}, c_{4}^{2}=\frac{\gamma}{\rho j}, \omega^{*}=\frac{\rho c_{1}^{2} C_{e}}{K}, \varepsilon=\frac{\beta_{0}^{2} \theta_{0}}{\rho^{2} c_{1}^{2} C_{e}}, \delta^{2}=\frac{c_{2}^{2}}{c_{1}^{2}} .
$$

Here a comma in the subscript of a variable denotes the derivative with respect to space and dot denotes the derivative with respect to time.

In terms of potential functions $\psi_{1}$ and $\psi_{2}$, given by

$$
\begin{aligned}
& u_{1}=\psi_{1,1}+\psi_{2,2} \\
& u_{2}=\psi_{1,2}-\psi_{2,1},
\end{aligned}
$$


equations (3.1)-(3.4) will become,

$$
\begin{gathered}
\left(\nabla^{2}-\frac{d^{2}}{d t^{2}}\right) \psi_{1}-\theta=0 \\
\left(\delta^{2} \nabla^{2}-\frac{d^{2}}{d t^{2}}\right) \psi_{2}+\phi=0 \\
\delta_{1}^{2} \nabla^{2} \phi-\delta_{2}^{2} \nabla^{2} \psi_{2}-2 \phi=\frac{\omega^{* 2} \rho j}{\kappa} \ddot{\phi} \\
\nabla^{2} \theta=\left(1+\tau \frac{\partial}{\partial t}\right) \dot{\theta}+\varepsilon\left(1+\tau \frac{\partial}{\partial t}\right) \nabla^{2} \dot{\psi}_{1}
\end{gathered}
$$

in which $\delta_{1}^{2}=\frac{\gamma \omega^{* 2}}{\kappa c_{1}^{2}}, \delta_{2}^{2}=\frac{\kappa}{\rho c_{1}^{2}}$.

\section{Solution of the problem and boundary conditions}

The solutions of the equations (3.5)-(3.8) can be obtained in the following form:

$$
\begin{gathered}
\psi_{1}=\left[A \sin \left(m_{1} x_{2}\right)+B \cos \left(m_{1} x_{2}\right)+C \sin \left(m_{2} x_{2}\right)+D \cos \left(m_{2} x_{2}\right)\right] e^{i \xi\left(x_{1}-c t\right)} \\
\begin{array}{r}
\psi_{2}=\left[E \sin \left(m_{3} x_{2}\right)+F \cos \left(m_{3} x_{2}\right)+G \sin \left(m_{4} x_{2}\right)+H \cos \left(m_{4} x_{2}\right)\right] e^{i \xi\left(x_{1}-c t\right)} \\
\theta=\left(\alpha^{2}-m_{1}^{2}\right)\left[A \sin \left(m_{1} x_{2}\right)+B \cos \left(m_{1} x_{2}\right)\right] e^{i \xi\left(x_{1}-c t\right)} \\
+\left(\alpha^{2}-m_{2}^{2}\right)\left[C \sin \left(m_{2} x_{2}\right)+D \cos \left(m_{2} x_{2}\right)\right] e^{i \xi\left(x_{1}-c t\right)} \\
\phi=\delta^{2}\left(m_{3}^{2}-\beta^{2}\right)\left[E \sin \left(m_{3} x_{2}\right)+F \cos \left(m_{3} x_{2}\right)\right] e^{i \xi\left(x_{1}-c t\right)} \\
+\delta^{2}\left(m_{4}^{2}-\beta^{2}\right)\left[G \sin \left(m_{4} x_{2}\right)+H \cos \left(m_{4} x_{2}\right)\right] e^{i \xi\left(x_{1}-c t\right)}
\end{array}
\end{gathered}
$$

where $\alpha^{2}=\xi^{2}\left(c^{2}-1\right), \beta^{2}=\xi^{2}\left(\frac{c^{2}}{\delta^{2}}-1\right), m_{i}^{2}=\xi^{2}\left(a_{i}^{2} c^{2}-1\right), i=1,2,3,4$

$$
\begin{gathered}
a_{1}^{2}, a_{2}^{2}=\left[\frac{\left(1+\tau^{\prime}+\varepsilon \tau^{\prime}\right) \pm \sqrt{\left(1+\tau^{\prime}+\varepsilon \tau^{\prime}\right)^{2}-4 \tau^{\prime}}}{2}\right], \\
a_{3}^{2}, a_{4}^{2}=\left[\frac{\left(\delta_{1}^{2}+\delta_{2}^{2} \omega^{-2}+\delta^{2} \tau^{\prime \prime}\right) \pm \sqrt{\left(\delta_{1}^{2}+\delta_{2}^{2} \omega^{-2}+\delta^{2} \tau^{\prime \prime}\right)^{2}-4 \tau^{\prime \prime}}}{2 \delta^{2} \delta_{1}^{2}}\right], \\
\tau^{\prime}=\left(\tau+i \omega^{-1}\right), \tau^{\prime \prime}=\left(\frac{\omega^{* 2} \rho j}{\kappa}-2 \omega^{-2}\right) .
\end{gathered}
$$


The non-dimension mechanical boundary conditions at the boundaries $x_{2}= \pm h$ are given by

and thermal condition

$$
\begin{gathered}
u_{2,2}+\frac{\lambda}{\rho c_{1}^{2}} u_{1,1}-\theta=0 \\
\delta^{2} u_{1,2}+\frac{\mu}{\rho c_{1}^{2}} u_{2,1}+\phi=0 \\
\varphi, 2=0
\end{gathered}
$$

$$
\theta, 2+h_{1} \theta=0
$$

where $h_{1}$ is the surface heat transfer co-efficient and $h_{1} \rightarrow \infty$ when the boundaries are isothermal where as $h_{1} \rightarrow 0$ when the boundaries are insulated.

\section{Derivation of secular equations}

\subsection{Symmetric vibration}

For symmetric vibration, using the boundary conditions, we obtain four simultaneous, homogeneous, linear equations with four unknowns B, D, E, G and for non-trivial solutions of the system, we obtain the following dispersive equations for micropolar thermoelastic media, where the effect of thermal relaxation is incorporated:

$$
\begin{aligned}
& {\left[\left(\beta^{2}-m_{3}^{2}\right) m_{3} \tan \left(m_{4} h\right)-\left(\beta^{2}-m_{4}^{2}\right) m_{4} \tan \left(m_{3} h\right)\right]} \\
& \quad \times\left[\frac{1}{\left[\tan \left(m_{1} h\right)\right]^{ \pm 1}}-\frac{\left(\alpha^{2}-m_{1}^{2}\right)}{\left(\alpha^{2}-m_{2}^{2}\right)}\left[\frac{m_{1}}{m_{2} \tan \left(m_{2} h\right)}\right]^{ \pm 1}\right]^{ \pm 1} \\
& \quad=\frac{\left(\frac{2 \mu+\kappa}{\rho c_{1}^{2}}\right)^{2}}{\left(c^{2}-\frac{2 \mu+\kappa}{\rho c_{1}^{2}}\right)} \frac{m_{1} m_{3} m_{4}\left(m_{4}^{2}-m_{3}^{2}\right)}{\delta^{2}\left(\beta^{2}-\xi^{2}\left(1-\frac{\delta_{2}^{2}}{\delta^{2}}\right)\right)}\left[\frac{\left(m_{2}^{2}-m_{1}^{2}\right)}{\left(\alpha^{2}-m_{2}^{2}\right)}\right]^{ \pm 1} .
\end{aligned}
$$

\subsection{Anti-symmetric vibration}

Similarly, the dispersive equations for anti-symmetric vibration are given by

$$
\begin{aligned}
& {\left[\left(\beta^{2}-m_{3}^{2}\right) \frac{m_{3}}{\tan \left(m_{4} h\right)}-\left(\beta^{2}-m_{4}^{2}\right) \frac{m_{4}}{\tan \left(m_{3} h\right)}\right]} \\
& \quad \times\left[\left[\tan \left(m_{1} h\right)\right]^{ \pm 1}-\frac{\left(\alpha^{2}-m_{1}^{2}\right)}{\left(\alpha^{2}-m_{2}^{2}\right)}\left[\frac{m_{1} \tan \left(m_{2} h\right)}{m_{2}}\right]^{ \pm 1}\right]^{ \pm 1} \\
& =\frac{\left(\frac{2 \mu+\kappa}{\rho c_{1}^{2}}\right)^{2}}{\left(c^{2}-\frac{2 \mu+\kappa}{\rho c_{1}^{2}}\right)} \frac{m_{1} m_{3} m_{4}\left(m_{4}^{2}-m_{3}^{2}\right)}{\delta^{2}\left(\beta^{2}-\xi^{2}\left(1-\frac{\delta_{2}^{2}}{\delta^{2}}\right)\right)}\left[\frac{\left(m_{2}^{2}-m_{1}^{2}\right)}{\left(\alpha^{2}-m_{2}^{2}\right)}\right]^{ \pm 1},
\end{aligned}
$$

where ' + ' sign corresponds to the insulated boundaries and ' - ' sign refers to the isothermal boundaries. 
In particular, if the micropolar effect is absent; we have

$$
a_{3}^{2}=\frac{1}{\delta^{2}} \text { and } \beta^{2}=m_{3}^{2}=\xi^{2}\left(\frac{c^{2}}{\delta^{2}}-1\right) .
$$

Thus from the equations (5.1)-(5.2), we obtain

$$
\left[\frac{\tan \left(m_{1} h\right)}{\tan (\beta h)}\right]^{ \pm 1}-\frac{m_{1}\left(\alpha^{2}-m_{1}^{2}\right)}{m_{2}\left(\alpha^{2}-m_{2}^{2}\right)}\left[\frac{\tan \left(m_{2} h\right)}{\tan (\beta h)}\right]^{ \pm 1}=\frac{4 \xi^{2} m_{1} \beta\left(m_{2}^{2}-m_{1}^{2}\right)}{\left(\xi^{2}-\beta^{2}\right)^{2}\left(\alpha^{2}-m_{2}^{2}\right)},
$$

for insulated boundaries and

$$
\left[\frac{\tan (\beta h)}{\tan \left(m_{1} h\right)}\right]^{ \pm 1}-\frac{m_{2}\left(\alpha^{2}-m_{1}^{2}\right)}{m_{1}\left(\alpha^{2}-m_{2}^{2}\right)}\left[\frac{\tan (\beta h)}{\tan \left(m_{2} h\right)}\right]^{ \pm 1}=\frac{\left(\xi^{2}-\beta^{2}\right)^{2}\left(m_{2}^{2}-m_{1}^{2}\right)}{4 \xi^{2} m_{1} \beta\left(\alpha^{2}-m_{2}^{2}\right)},
$$

for isothermal boundaries, where ' + ' sign refers to the anti-symmetric vibration and '- 'sign refers to the symmetric vibration. It is seen that the equations (5.3) and (5.4) are similar to the equations obtained by Sharma (2001).

Further, substituting $\alpha=m_{1}$ e obtain from equations (5.3) and (5.4) the Rayleigh-Lamb equation, as in Graff (1991)

$$
\frac{\tan (\alpha h)}{\tan (\beta h)}=-\left[\frac{4 \xi^{2} \alpha \beta}{\left(\xi^{2}-\beta^{2}\right)^{2}}\right]^{ \pm 1}
$$

Thus equations (5.1) and (5.2) are more general dispersive equations for symmetric and anti symmetric mode of vibrations respectively, which includes the effect of micropolar as well as thermal relaxation of time.

\section{Discussion of secular equations}

In this section, our discussion is devoted on different aspects of phase velocity, specially when the value of the phase velocity 'c' lies on different regions.

\subsection{Region-I}

When wave is propagating with the phase velocity characterized by $c<\delta$, replacing $\frac{\tanh z}{z}$ by $\frac{\tan z^{\prime}}{z^{\prime}}$, i $\alpha$ by $\alpha^{\prime}$ and $i \beta$ by $\beta^{\prime}$, omitting the primes, we obtain from the equations (5.1)-(5.2)

$$
\begin{aligned}
& {\left[\left(\beta^{2}-m_{3}^{2}\right) m_{3} \tanh \left(m_{4} h\right)-\left(\beta^{2}-m_{4}^{2}\right) m_{4} \tanh \left(m_{3} h\right)\right]} \\
& \quad \times\left[\frac{1}{\left[\tanh \left(m_{1} h\right)\right]^{ \pm 1}}-\frac{\left(\alpha^{2}-m_{1}^{2}\right)}{\left(\alpha^{2}-m_{2}^{2}\right)}\left[\frac{m_{1}}{m_{2} \tanh \left(m_{2} h\right)}\right]^{ \pm 1}\right]^{ \pm 1} \\
& \quad=-\frac{\left(\frac{2 \mu+\kappa}{\rho c_{1}^{2}}\right)^{2}}{\left(c^{2}-\frac{2 \mu+\kappa}{\rho c_{1}^{2}}\right)} \frac{m_{1} m_{3} m_{4}\left(m_{4}^{2}-m_{3}^{2}\right)}{\delta^{2}\left(\beta^{2}-\xi^{2}\left(1-\frac{\delta_{2}^{2}}{\delta^{2}}\right)\right)}\left[\frac{\left(m_{2}^{2}-m_{1}^{2}\right)}{\left(\alpha^{2}-m_{2}^{2}\right)}\right]^{ \pm 1},
\end{aligned}
$$


for symmetric vibration and

$$
\begin{aligned}
& {\left[\left(\beta^{2}-m_{3}^{2}\right) \frac{m_{3}}{\tanh \left(m_{4} h\right)}-\left(\beta^{2}-m_{4}^{2}\right) \frac{m_{4}}{\tanh \left(m_{3} h\right)}\right]} \\
& \quad \times\left[\left[\tanh \left(m_{1} h\right)\right]^{ \pm 1}-\frac{\left(\alpha^{2}-m_{1}^{2}\right)}{\left(\alpha^{2}-m_{2}^{2}\right)}\left[\frac{m_{1} \tanh \left(m_{2} h\right)}{m_{2}}\right]^{ \pm 1}\right]^{ \pm 1} \\
& \quad=-\frac{\left(\frac{2 \mu+\kappa}{\rho c_{1}^{2}}\right)^{2}}{\left(c^{2}-\frac{2 \mu+\kappa}{\rho c_{1}^{2}}\right)} \frac{m_{1} m_{3} m_{4}\left(m_{4}^{2}-m_{3}^{2}\right)}{\delta^{2}\left(\beta^{2}-\xi^{2}\left(1-\frac{\delta_{2}^{2}}{\delta^{2}}\right)\right)}\left[\frac{\left(m_{2}^{2}-m_{1}^{2}\right)}{\left(\alpha^{2}-m_{2}^{2}\right)}\right]^{ \pm 1},
\end{aligned}
$$

for anti-symmetric vibration, where ' + ' sign corresponds the insulated boundaries and '-' sign refers to the isothermal boundaries.

For long wave length in symmetric vibration, replacing tanh $z$ by $z$ we obtain from the equations (6.1)-(6.2),

$$
\left(c^{2}-\frac{2 \mu+\kappa}{\rho c_{1}^{2}}\right)\left(\alpha^{2}-m_{1}^{2}-m_{2}^{2}\right)=\left(\frac{2 \mu+\kappa}{\rho c_{1}^{2}}\right)^{2} \frac{m_{1}^{2} m_{2}^{2}}{\delta^{2}\left(\beta^{2}-\xi^{2}\left(1-\frac{\delta_{2}^{2}}{\delta^{2}}\right)\right)},
$$

in the case of insulated boundaries, and

$$
c^{2}=\frac{(2 \lambda+2 \mu+\kappa)(2 \mu+\kappa)}{(\lambda+2 \mu+\kappa)^{2}}
$$

in the case of isothermal boundaries. It is seen from equation (6.4) that for long wave length, the phase velocity agrees with the result obtained by Eringen (1998), which implies that there is no effect of temperature for the case of long wave length.

Similarly, for long wave length in anti-symmetric vibration, replacing $\frac{\tanh z}{z}$ by $\left(1-\frac{z^{2}}{3}\right)$ we obtain,

$$
\begin{aligned}
& {\left[\frac{\left(\beta^{2}-m_{3}^{2}\right)}{m_{4}^{2}\left(1-\frac{m_{4}^{2} h^{2}}{3}\right)}-\frac{\left(\beta^{2}-m_{4}^{2}\right)}{m_{3}^{2}\left(1-\frac{m_{3}^{2} h^{2}}{3}\right)}\right]\left[\left(1-\frac{m_{1}^{2} h^{2}}{3}\right)^{ \pm 1}-\frac{\left(\alpha^{2}-m_{1}^{2}\right)}{\left(\alpha^{2}-m_{2}^{2}\right)}\left(1-\frac{m_{2}^{2} h^{2}}{3}\right)^{ \pm 1}\right]^{ \pm 1}} \\
& =-\frac{\left(\frac{2 \mu+\kappa}{\rho c_{1}^{2}}\right)^{2}}{\left(c^{2}-\frac{2 \mu+\kappa}{\rho c_{1}^{2}}\right)} \frac{\left(m_{4}^{2}-m_{3}^{2}\right)}{\delta^{2}\left(\beta^{2}-\left(1-\frac{\delta_{2}^{2}}{\delta^{2}}\right)\right)}\left[\frac{\left(m_{2}^{2}-m_{1}^{2}\right)}{\left(\alpha^{2}-m_{2}^{2}\right)}\right]^{ \pm 1}
\end{aligned}
$$

where ' + ' sign corresponds to the insulated boundaries and ' - ' sign refers to the isothermal boundaries.

For short wave length in symmetric vibration replacing tanh $z$ by 1 , we obtain from the secular equation (6.1)

$$
\begin{aligned}
& {\left[m_{4}^{2}+m_{3}^{2}-m_{3} m_{4}-\beta^{2}\right] \frac{\left(c^{2}-\frac{2 \mu+\kappa}{\rho c_{1}^{2}}\right)}{\left(\frac{2 \mu+\kappa}{\rho c_{1}^{2}}\right)^{2}} \frac{\delta^{2}\left(\beta^{2}-\left(1-\frac{\delta_{2}^{2}}{\delta^{2}}\right)\right)}{m_{3} m_{4}\left(m_{3}+m_{4}\right)}} \\
& \quad=-\left\{\frac{m_{1} m_{2}\left(m_{1}+m_{2}\right)}{\left[m_{1}^{2}+m_{2}^{2}-m_{1} m_{2}-\alpha^{2}\right]}, \frac{\left(\alpha^{2}+m_{1} m_{2}\right)}{\left(m_{1}+m_{2}\right)}\right\},
\end{aligned}
$$


where the first term in the right hand side of the equality sign corresponds to the secular equation for insulated boundaries and second term for isothermal boundaries. For anti-symmetric vibration in short wave length, we obtain the same equation as that of symmetric vibration.

\subsection{Region-II}

When the phase velocity of the wave propagation ' $\mathrm{c}$ ' is characterized by $\delta<c<1$, in this case replacing $\alpha^{\prime}=i \alpha, m_{1}^{\prime}=i m_{1}, m_{2}^{\prime}=i m_{2}$ from the equations (5.1)-(5.2) by omitting the primes, we obtain,

$$
\begin{aligned}
& {\left[\left(\beta^{2}-m_{3}^{2}\right) m_{3} \tan \left(m_{4} h\right)-\left(\beta^{2}-m_{4}^{2}\right) m_{4} \tan \left(m_{3} h\right)\right]} \\
& \quad \times\left[\frac{1}{\left[\tanh \left(m_{1} h\right)\right]^{ \pm 1}}-\frac{\left(\alpha^{2}-m_{1}^{2}\right)}{\left(\alpha^{2}-m_{2}^{2}\right)}\left[\frac{m_{1}}{m_{2} \tanh \left(m_{2} h\right)}\right]^{ \pm 1}\right]^{ \pm 1} \\
& \quad=-\frac{\left(\frac{2 \mu+\kappa}{\rho c_{1}^{2}}\right)^{2}}{\left(c^{2}-\frac{2 \mu+\kappa}{\rho c_{1}^{2}}\right)} \frac{m_{1} m_{3} m_{4}\left(m_{4}^{2}-m_{3}^{2}\right)}{\delta^{2}\left(\beta^{2}-\xi^{2}\left(1-\frac{\delta_{2}^{2}}{\delta^{2}}\right)\right)}\left[\frac{\left(m_{2}^{2}-m_{1}^{2}\right)}{\left(\alpha^{2}-m_{2}^{2}\right)}\right]^{ \pm 1}
\end{aligned}
$$

for symmetric vibration, and

$$
\begin{aligned}
& {\left[\left(\beta^{2}-m_{3}^{2}\right) \frac{m_{3}}{\tan \left(m_{4} h\right)}-\left(\beta^{2}-m_{4}^{2}\right) \frac{m_{4}}{\tan \left(m_{3} h\right)}\right]} \\
& \quad \times\left[\left[\tanh \left(m_{1} h\right)\right]^{ \pm 1}-\frac{\left(\alpha^{2}-m_{1}^{2}\right)}{\left(\alpha^{2}-m_{2}^{2}\right)}\left[\frac{m_{1} \tanh \left(m_{2} h\right)}{m_{2}}\right]^{ \pm 1}\right]^{ \pm 1} \\
& \quad=\frac{\left(\frac{2 \mu+\kappa}{\rho c_{1}^{2}}\right)^{2}}{\left(c^{2}-\frac{2 \mu+\kappa}{\rho c_{1}^{2}}\right)} \frac{m_{1} m_{3} m_{4}\left(m_{4}^{2}-m_{3}^{2}\right)}{\delta^{2}\left(\beta^{2}-\xi^{2}\left(1-\frac{\delta_{2}^{2}}{\delta^{2}}\right)\right)}\left[\frac{\left(m_{2}^{2}-m_{1}^{2}\right)}{\left(\alpha^{2}-m_{2}^{2}\right)}\right]^{ \pm 1}
\end{aligned}
$$

for anti-symmetric vibration

\subsection{Region-III}

This region is characterized by the value of the phase velocity $c>1$. In this case the secular equations are given by the equations (5.1)-(5.2).

\section{Lame mode}

For convenience of writing $\beta^{2}=\xi^{2}\left(1-\frac{\delta_{2}^{2}}{\delta^{2}}\right)$, we obtain the following frequency equation,

$$
c= \pm \frac{1}{a_{3}} \sqrt{\frac{n^{2} \pi^{2}}{4 \xi^{2} h^{2}}+1}, n=0,1,2,3 \ldots
$$

which is the Lame modes, a special class of exact solution, first identified by Lamb (1917). 
Now in the absence of micropolar effect, $a_{3}^{2}=\frac{1}{\delta^{2}}, \beta^{2}=m_{3}^{2}=\xi^{2}\left(\frac{c^{2}}{\delta^{2}}-1\right)$, and consequently the frequency equation is given by

$$
c= \pm \delta \sqrt{\frac{n^{2} \pi^{2}}{4 \xi^{2} h^{2}}+1}, n=0,1,2,3 \ldots
$$

\section{Numerical analysis}

With the view of illustrating the different theoretical results in the preceding sections and comparing these with various theories of classical as well as micropolar thermoelasticity. We now present some numerical results for this purpose and we select aluminium-epoxy composite, the physical data for this material are given as in (Singh \& Kumar 1998),

$$
\begin{gathered}
\lambda=7.59 \times 10^{10} \mathrm{Nm}^{-2}, \mu=1.89 \times 10^{10} \mathrm{Nm}^{-2}, \kappa=0.0149 \times 10^{10} \mathrm{Nm}^{-2} \\
K=2.508 \mathrm{~K} / \mathrm{m} \mathrm{sec}{ }^{\circ} \mathrm{C}, \rho=2.19 \times 10^{3} \mathrm{~kg} \mathrm{~m}^{-3}, C_{e}=961.4 \mathrm{Jg}^{-1} /{ }^{\circ} \mathrm{C} \\
\gamma=0.0268 \times 10^{10} \mathrm{Nm}^{-2}, \theta_{0}=23^{\circ} \mathrm{C}, \tau=6.131 \times 10^{-13} \mathrm{~s} \\
h=1.0, \omega^{*}=4.666 \times 10^{11} \mathrm{~s}^{-1} .
\end{gathered}
$$

The non-dimensional phase velocities and attenuation coefficients of symmetric as well as anti-symmetric modes of wave propagation have been computed for various values of nondimensional wave numbers from the secular equations (5.1) and (5.2). The corresponding numerical results are presented graphically in different modes $(n=0,1,2,3)$ as a function of non-dimensional wave number in figures 1 and 2 for generalized micropolar thermoelasticity (GMTE) in comparison with generalized thermoelasticity (GTE) as well as classical thermoelasticity (CTE). The changes of non-dimensional attenuation coefficients with non-dimensional wave numbers in different modes $(\mathrm{n}=0,1,2,3)$ for symmetric and anti-symmetric vibrations are exhibited in figures 3 and 4.

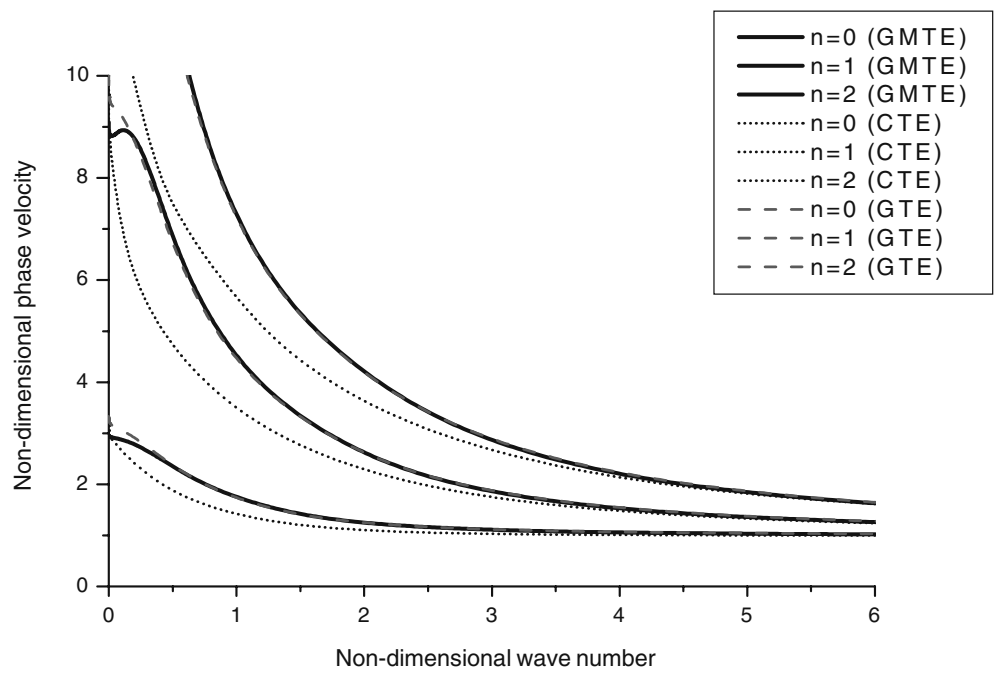

Figure 1. Dispersion curve for symmetric mode. 


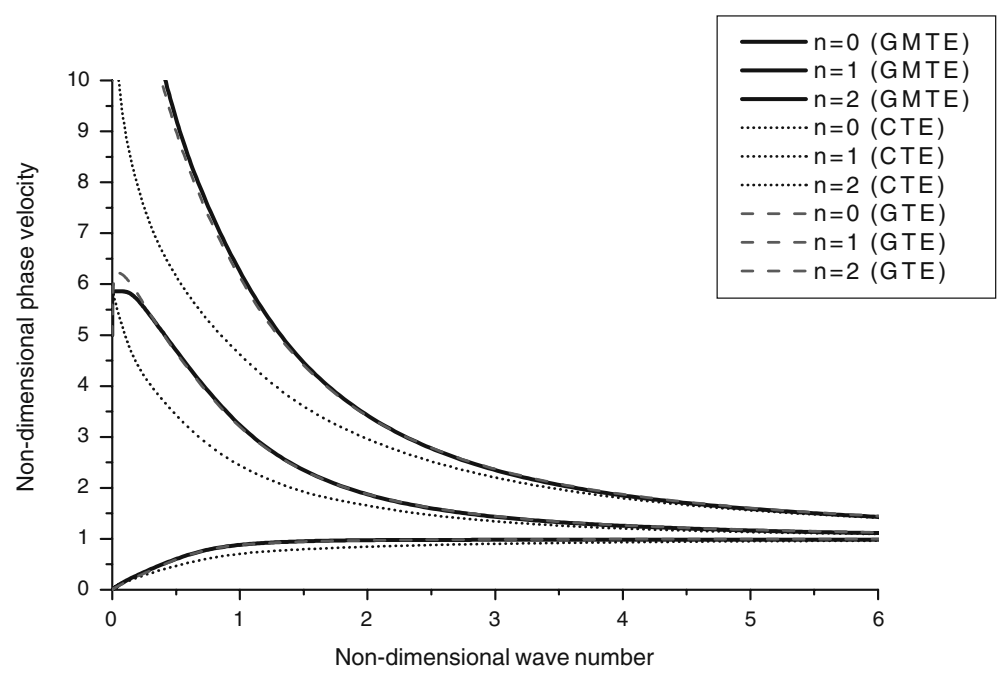

Figure 2. Dispersion curve for anti-symmetric mode.

It is observed that the phase velocity of lowest symmetric mode $(\mathrm{n}=0)$ decreases from a higher value than 2 at vanishing wave number and becomes steady with increasing of wave numbers, where as in anti-symmetric mode it increases from zero and becomes steady. The phase velocities for higher modes of wave propagation attains larger values than the lowest mode at vanishing wave number then rapidly converges towards the lowest mode of wave propagation and become steady with increasing wave number. It is also observed that the natures of phase velocities are quite similar for symmetric and anti-symmetric modes with increasing wave number. The reason for this type of asymptotic approach of phase velocities is that for small wave lengths (or high frequencies) the material plate behaves like a thick slab and hence the coupling

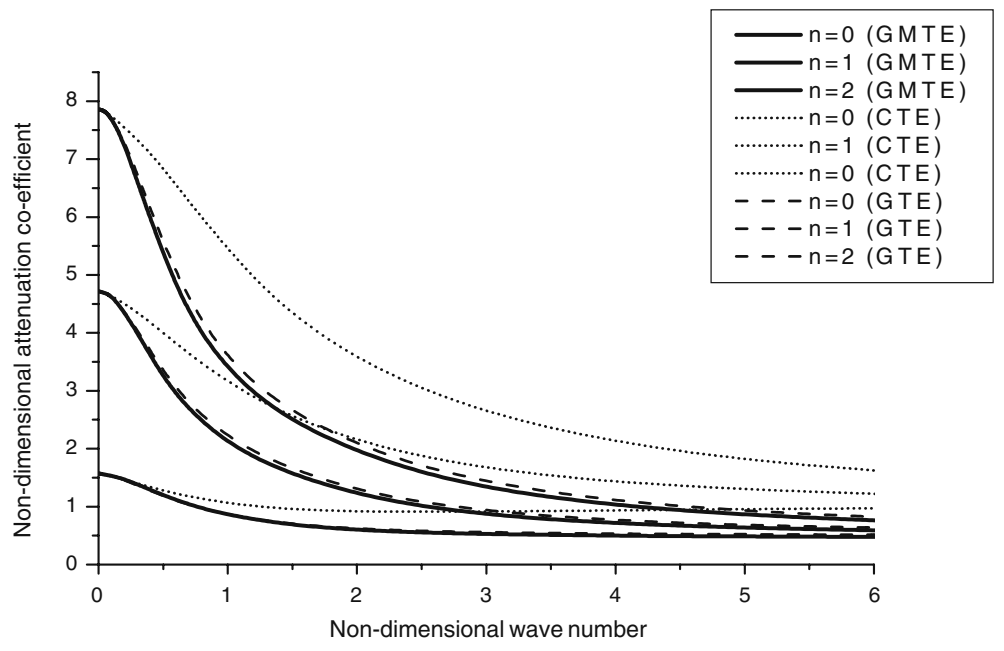

Figure 3. Dispersion curve for symmetric mode. 


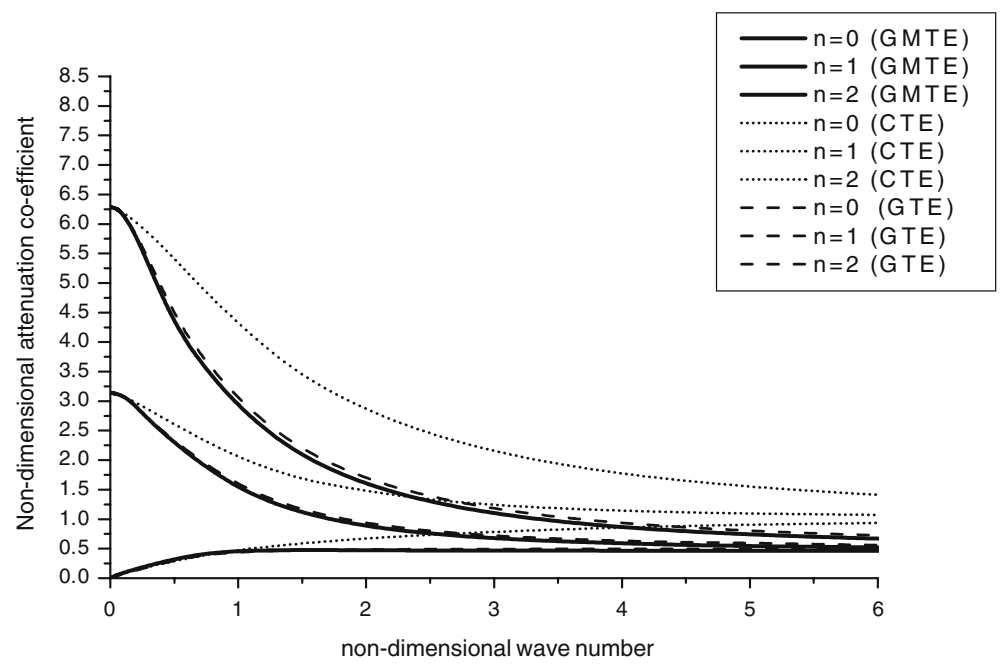

Figure 4. Dispersion curve for anti-symmetric mode.

between the lower and upper surfaces is reduced and as a result the nature of symmetric and anti-symmetric waves become more and more similar.

In comparisons with GTE and CTE it has been seen that the phase velocities of CTE attains smaller values than that of GMTE for small wave length and similar with increasing of wave number whereas the wave profile of GTE is almost same as GMTE throughout, expect in the range 0 to 0.33 of non-dimensional wave number where the phase velocity is larger than that of micropolar elasticity for symmetric vibration only.

From the figures 3 and 4 it is observed that in symmetric vibration the values of the attenuation coefficient decreases from a value greater than 1 whereas in anti-symmetric vibration, attenuation coefficient increases its value from zero in the neighborhood of $\xi=0$ for the lowest mode $(n=0)$ of wave propagation. After that the attenuation coefficient profile becomes steady for $n=0$. In higher modes of wave propagation, the profiles of attenuation coefficient sharply slash down from higher values and converge towards the lowest mode of wave propagation. The values of attenuation coefficient satisfy the inequality $C T E>C T E>G M T E$ for all values of non-dimensional wave number except at $\xi=0$.

\section{References}

Boley B A, Weiner J H 1960 Theory of thermal stresses. New York: John Wiley and Sons

Cattanco C 1958 A form of heat equation which eliminates the paradox of instantaneous propagation. $C$. R. Acad. Sci. 24: 7431-433

Chandrasekharaiah D S 1986 Thermoelasticity with second sound: A review. Appl. Mech. Rev. 39: 355-376

Chandrasekharaiah D S, Debnath L 1988 Magneto-thermoelastic disturbance with thermal relaxation in a solid due to heat sources. Comput. Math. Appl. 15: 483-490

Chowdhury K L, Glockner P G 1979 On thermorigid dielectrics. J. Thermal Stress. 2: 73-95

Chowdhury K L, Glockner P G, Epstien M 1979 On the thermodynamics of nonlinear elastic dielectrics. Int. J. Non-Linear Mech. 13: 311-322

Das N C, Lahiri A, Giri R R 1997 Eigenvalue approach to generalized thermoelasticity. Indian J. Pure Appl. Math. 28: 1573-1594 
Dhaliwal R S, Sherief H H 1980 Generalized thermoelasticity for anisotropic media. Quart. Appl. Math. 38: $1-8$

Erbay S, Suhubi E S 1989 Non linear wave propagation in micropolar media - I and I. Int. J. Eng. Sci. 27: 895-920

Eringen A C 1965 Theory of micropolar continua. In Proceedings of the Ninth Midwestern Mechanics Conference, Wisconsin, 16-18 August, New York: Wiley

Eringen A C 1966 Linear theory of micropolar elasticity. J. Math. Mech. 15: 909-923

Eringen A C 1973 Linear theory of non-local microelasticity and dispersion of plane waves. Lett. Appl. Eng. Sci. 1: 11-17

Eringen A C 1998 Microcontinuum field theories: Foundation and solids. New York: Springer

Eringen A C, Suhubi E S 1964 Nonlinear theory of simple microelastic solids I \& II. Int. J. Eng. Sci. 2: 189-203, 389-404

Eringen A C, Maugin G A 1990 Electrodynamics of continua. New York: Springer Verlag

Ezzat M A, Othman M I, EI-Karamany A M S 2002 State space approach to two-dimensional generalized thermo-viscoelasticity media. J. Therm. Stress. 25(6): 507-522

Graff K F 1991 Wave motion in elastic solids. New York: Dover Publications, Inc

Green A E, Laws N 1972 On the entropy production inequality. Arch. Ration. Mech. Anal. 45: 47-53

Green A E, Lindsay K A 1972 Thermoelasticity. J. Elast. 2: 1-7

Green A E, Nagdi P M 1993 Thermoelasticity without energy dissipation. J. Elast. 31: 189-208

Iesan D 1967 On the linear theory of micropolar elasticity. Int. J. Eng. Sci. 7: 271-274

Kaliski S 1965 Wave equation of thermoelasticity. Bull. Acad. Polon. Sci. Série Sci. Technol. 13: 253-260

Kumar R, Ailawalia P 2006 Deformation due to time harmonic sources in micropolar thermoelastic medium possessing cubic symmetry with two relaxation times. Appl. Math. Mech. (English Edition) 27: 781-792

Kumar R, Partap G 2006 Rayleigh Lamb waves in micropolar isotropic elastic plate. Appl. Math. Mech. (English Edition) 27: 1049-1059

Kumar R, Ailawalia P 2007 Mechanical/thermal sources in a micropolar thermoelastic medium possessing cubic symmetry without energy dissipation. Int. J. Thermophys. 28: 342-367

Kumar R, Kansal T 2008 Propagation of Rayleigh waves on free surface of transversely isotropic generalized thermoelastic diffusion. Appl. Math. Mech. (English Edition) 29: 1451-1462

Lamb H 1917 On waves in an elastic plate. J. Phil Trans. Roy Soc. London, Série A 93: 114-128

Lord H W, Shulman Y 1967 A generalized dynamical theory of thermoelasticity. J. Mech. Phys. Solid 15: 299-309

McCarthy M F 1972 Wave propagation on generalized thermoelasticity. Int. J. Eng. Sci. 10: 593-602

Mukhopadhyay B, Bera R K 1992 Effect of thermal relaxation on electro-magnetic-thermo-visco-elastic plane waves in rotating media. Int. J. Eng. Sci. 30(3): 359-369

Muller J M 1967 On the entropy inequality. J. Arch. Ration. Mech. Analysis 26: 118-141

Muller J M 1971 The coldness of universal function in thermoelastic bodies. J. Arch. Ration. Mech. Anul. 41: 319-332

Nowacki W 1962 Thermoelasticity. London: Pergamon Press

Nowacki W 1970 Theory of micropolar elasticity. Udline Course \& Lectures No. 25. Wien: Springer-Verlag

Nowacki W 1975 Dynamic problems of thermoelasticity, Leyden. The Netherlands: Noordhoff International Publishing

Nowinski J L 1978 Theory of thermoelasticity with applications, Leyden. The Netherlands: Sijthoff \& Noordhoff International Publishers

Othman M I A, Singh G B 2007 The effect of rotation on generalized micropolar thermoelasticity for a half-space under five theories. Int. J. Solids Struct. 44: 2748-2762

Rakshit M, Mukhopadhyay B 2005 An electro-magneto-thermo-visco-elastic problem in an infinite medium with a cylindrical hole. Int. J. Eng. Sci. 43: 925-936

Rakshit M, Mukhopadhyay B 2007 A two-dimensional thermoviscoelastic problem due to instantaneous point heat sources. Math. Comput. Model. 46: 1388-1397

Rao K M 1988 Longitudinal wave propagation in a micropolar wave guide. Int. J. Eng. Sci. 26: 135-141 
Sharma J N 2001 On the propagation of thermoelastic waves in homogeneous isotropic plates. Indian J. Pure Appl. Math. 32: 1329-1341

Singh B, Kumar R 1998 Wave propagation in a generalized thermo-microstretch elastic solid. Int. J. Eng. Sci. 36: 891-912

Suhubi E S 1982 A generalized theory of simple thermomechanical materials. Int. J. Eng. Sci. 20: 367-371

Suhubi E S, Eringen A C 1964 Nonlinear theory of simple microelastic solids II. Int. J. Eng. Sci. 2: 389-404

Tomar S K, Kumar R, Kaushik V P 1998 Wave propagation of micropolar elastic medium with stretch. Int. J. Eng. Sci. 36: 683-698 